\title{
La creatividad del reportero en zonas conflictivas de América Latina
}

\author{
Víctor CERDÁN MARTÍNEZ* \\ victorcerdanmartinez@yahoo.es
}

(Abstracts y palabras clave al final del artículo)

Enviado: 5 de septiembre de 2011

Evaluado: 17 de febrero de 2012

Aceptado: 25 de abril de 2012

\section{INTRODUCCIÓN}

Lo inestable se forja en la imaginación, el lugar donde el periodista creativo viaja antes de enfrentarse a la realidad. El reportero imagina posibles imprevistos para adelantarse a los problemas que puedan suceder cuando va a retratar barrios con altos índices de violencia. Según Sternberg (1999), el profesional ha de viajar por la irrealidad como paso obligatorio para volver a la realidad misma, convirtiendo en posibilidades todo lo que se encuentre en el ambiente. Es imposible predecir el futuro, pero no el estar preparado para lo que pueda pasar. Aunque no existen soluciones universales, quiero resaltar en este artículo la importancia del pensamiento creativo en la profesión del reportero de televisión.

Cuando me enteré que Cuadernos de Información y Comunicación iba a realizar este número sobre periodismo profesional lo primero que pensé fue en el concepto de creatividad. Creatividad como proceso comunicativo que ayuda al reportero a retratar zonas donde es difícil y peligroso grabar. Pienso que la emisión de dichos reportajes en televisión genera un conocimiento por parte de los espectadores sobre la necesidad del desarrollo en los lugares que se retratan. Durante este proceso, el reportero, aunque siga un código deontológico y ético, puede que no cumpla uno de los requisitos que enuncia Randall (2008: 183) sobre el buen periodista: "no someter las informaciones a la aprobación ni al veto de personas ajenas al medio". Por ejemplo, en las favelas de Rio de Janeiro (Brasil) el reportero necesita el permiso de los narcotraficantes que mandan en cada barrio y estará expuesto al veto durante la grabación.

En este artículo analizo las estrategias creativas que he empleado como reportero para reflejar realidades sociales en zonas conflictivas de América Latina. Es un

\footnotetext{
${ }^{*}$ Doctor en Ciencias de la Información por la Universidad Complutense de Madrid.
} 
proceso comunicativo que comienza desde el pensamiento creativo del reportero. Es ahí donde voy a centrar el análisis de este artículo.

\section{METODOLOGÍA}

La palabra creatividad tiene un significado muy amplio y vago que incluye elementos de novedad, de creación e incluso de valor. Esta extensa definición abarca varios procesos, enteramente diferentes entre sí que en este artículo no podré abordar. Por ello, en lugar de hacer una inmensa recopilación de teorías sobre la creatividad voy a centrarme en ciertos enfoques que creo que son más útiles para el análisis de la labor del reportero en diversas zonas marginales. ¿Qué modelos de pensamiento impulsan la creatividad del reportero? ¿Qué representa lo que está normalizado? ¿Qué técnicas impulsan la creación de alternativas ante un imprevisto? Para Valbuena de la Fuente (1999), la creatividad es una aptitud o posibilidad de hacer algo nuevo y la creación es el resultado. Este proceso, según el autor, tiene tres principios básicos. Primero, es un trabajo individual. Segundo, el método o la acción sistemática (creática) ayuda más todavía que la imaginación al acto creativo. Tercero, el trabajo común puede ser un complemento, un estimulante y un apoyo para el acto creativo. A mi juicio, los dos autores que mejor desarrollan estas perspectivas son Ortega y Gasset y Edward de Bono. El primero desarrolla una teoría en la que diferencia la creación de ideas frente a la creencia en las ideas; "uno puede estar en las ideas o crear las ideas" (Ortega y Gasset 1945: 15). Por su parte, Edward de Bono, en su teoría sobre el pensamiento creativo, realiza una división entre el pensamiento lateral frente al pensamiento vertical. Según De Bono (1994: 95), el pensamiento lateral es el que permite aportar técnicas e instrumentos de trabajo que constituyen una manera sistemática de obtener nuevas ideas. El pensamiento vertical, en cambio, toma una posición y después trata de construir sobre esta una base lógica sin cuestionar el punto de partida.

El concepto de creencias de Ortega y Gasset se asemeja al pensamiento vertical de De Bono en que ambos modelos parten de ideas que no son sometidas a duda. Esto provoca respuestas eficaces, pero basadas en normas estandarizadas y por lo tanto anti-creativas. Sin embargo, cuando empleamos el pensamiento lateral o dudamos para crear nuevas ideas nos adentramos en el pensamiento creativo. Para Ortega y Gasset (1945: 34) las ideas pretenden sustituir el mundo inestable, ambiguo, de la duda, por un mundo en el que la ambigüedad desaparece. ¿Cómo se logra esto? El autor asegura que el camino consiste en inventar mundos. La idea es imaginación. "Al hombre no le es dado ningún mundo ya determinado. Sólo le son dadas las penalidades y las alegrías de su vida. Orientado por ellas, tiene que inventar el mundo". Para De Bono (1994: 108), la persona que quiere ser creativa tiene que tomar conciencia de la fluidez de la percepción y de la posibilidad de tener múltiples percepciones, todas ellas valiosas. Según el autor, el profesional tiene que remplazar el "es" por un "puede ser". Sin embargo, una vez terminado nuestro pensamiento creativo tenemos que volver al mundo de la lógica, para presentar ideas sólidas, factibles y de valor comprobable. 


\section{LOS DOS CAMINOS DEL REPORTERO}

Es indudable que cualquier reportero aporta una sensibilidad personal al afrontar un nuevo proyecto. Sin embargo, el profesional deberá escoger entre el camino de la creación de ideas o el camino de las creencias (el camino del pensamiento vertical o el del pensamiento lateral). "No se puede cavar un hoyo en un lugar diferente haciendo el mismo hoyo más profundo" (De Bono 1994: 96). Con esta descripción el autor pone énfasis sobre los diferentes enfoques y las diferentes maneras de ver las cosas. Si el periodista adopta el camino del pensamiento vertical, entonces aceptará los consejos de sus jefes y compañeros con más experiencia como válidos sin someterlos a juicio. Realizará un reportaje televisivo en base a esas normas, o lo que es lo mismo cavará el mismo hoyo, pero mas profundo.

El otro camino pretende cavar el mismo hoyo en otro lugar para luego perfeccionar el primer hoyo. Es el camino del pensamiento lateral. Según Ortega y Gasset (1945: 22), cuando creemos de verdad en una cosa no tenemos la "idea" de esa cosa, sino que simplemente "contamos con ella". El reportero que elija este camino no se limitará a contar con una idea preconcebida, sino que cuestionará los modelos anteriores. Explorará mundos imaginarios irracionales para luego volver a la lógica con ideas nuevas y útiles. Con este procedimiento el periodista se desplaza hacia los lados, para probar diferentes percepciones, diferentes conceptos, diferentes puntos de entrada. Usará diferentes métodos, incluidas las provocaciones, para salir de la línea habitual del pensamiento (Lipman 1991). Estos métodos pueden ser muy útiles en situaciones en las que el periodista tiene dificultades para mostrar una realidad social.

\section{OBJETO DE ESTUDIO}

En este artículo analizo mis percepciones como reportero en varias secuencias de reportajes que realicé en Latinoamérica. Dichos fragmentos corresponden a los programas de Callejeros, Viajeros ${ }^{1}$ : Rio de Janeiro y Montevideo. Los reportajes, de 50 minutos, fueron emitidos en prime time en el canal Cuatro en España en 2009 y 2011. El primero tuvo un $10 \%$ de share y 1.638 .000 espectadores, el segundo, un 8,6 y 1.634.000 seguidores. De hecho, según Castañares (2006: 100), la televisión es el más masivo de los medios de comunicación actuales, un poderoso venero del que continuamente surge un flujo discursivo muy heterogéneo. La emisión de estos programas, en el horario de máxima audiencia, contribuye al conocimiento de una serie de problemas sociales por un sector de la sociedad española. Voy a centrar este análisis en fragmentos de dos reportajes de Callejeros, Viajeros y en los procesos comunicativos que intervinieron en el desarrollo de los programas. Concretamente en el

${ }^{1}$ Callejeros, Viajeros es un programa de reportajes español que retrata lugares de todo el mundo desde diferentes perspectivas. El equipo que realiza los reportajes se compone únicamente de un operador de cámara y un reportero. 
estudio de mi percepción como reportero sobre los procesos creativos a la hora de realizar una denuncia social.

\section{ANÁLISIS}

La Favela Vigário Geral fue la primera que decidí grabar para el reportaje sobre Rio de Janeiro. Desde la producción del documental hasta la grabación opté por creer en una serie de ideas normalizadas en la profesión. Una de esas creencias es que si un reportero quiere retratar barrios conflictivos fuera de España debe contactar con ONGs o instituciones que trabajen en el lugar. Si decide no hacerlo de esta manera puede exponer la grabación al riesgo de perder la cámara o algo peor. Desde España pactamos con la ONG brasileña AfroReggae una serie de normas. Esas condiciones fueron impuestas por los narcotraficantes que gobiernan la favela, sin que pudiéramos negociar con ellos. Las restricciones principales eran dos: primero, guardar la cámara en la mochila a la entrada del barrio, y segundo, grabar las zonas de la favela y a las personas que ellos autorizaran durante el tiempo que consideraran oportuno.

A la entrada de la favela cruzamos una pasarela sobre la rua Bulhoes Marcial. Al final del recorrido (1.1), cuatro menores de edad vigilaban desde lo alto del puente la entrada a la favela. Ya habíamos guardado la cámara en la mochila. Los niños iban armados con escopetas, pistolas y fusiles de asalto. Hasta que no llegamos al edificio de la $\mathrm{ONG}$, dentro de la favela, no nos permitieron sacar la cámara. ¿Por qué no querían que les grabáramos con la cara difuminada? Por el simple hecho de que solicitaban dinero. Concretamente pedían 1.000 euros por grabar únicamente sus armas y 30.000 euros para poder retratar toda la favela con menos restricciones.
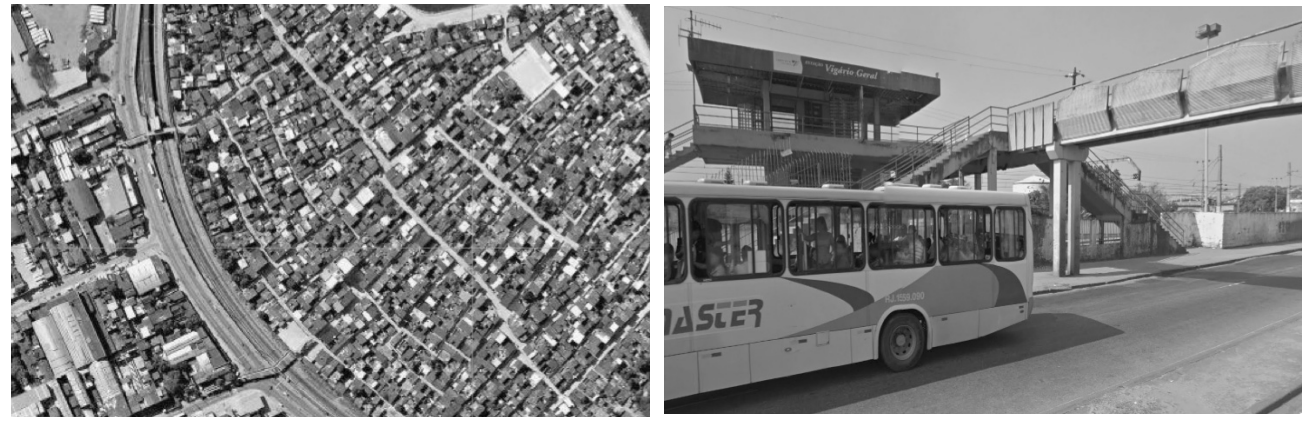

$(1.1)^{2}$

Como en Callejeros, Viajeros por norma nunca pagamos por conseguir información, decidí que la representante de la ONG, Eva Belenguer, nos narrara lo que había

\footnotetext{
2 Capturas digitales de Google Maps de la favela Vigário Geral.
} 
pasado al llegar a su oficina. Sin embargo, la voz en off no muestra la realidad, lo que hace es crear una referencia imaginaria donde el espectador puede percibir cierta inverosimilitud. De hecho, una de las claves del éxito de Callejeros 3 y Callejeros, Viajeros es la ausencia de voz en off. Lo que el reportero denuncia tiene que verse y contarse por parte de las víctimas y de los agresores a los que se entrevista. En Vigário Geral no pudimos reflejar en profundidad la realidad social del barrio. En el siguiente fragmento, expongo una parte del diálogo de dicha secuencia.

\section{SECUENCIA VIGÁRIO GERAL. RIO DE JANEIRO.}

REPORTERO: En 1993 se fundó AfroReggae, una asociación que pretende ayudar a los niños pobres de la favela a través de la música. Eva Belenguer trabaja con ellos. EVA: Vamos para Vigário Geral. La mayoría de las favelas son controladas por narcotraficantes, si por ejemplo estáis grabando narcotraficantes van a tener que quebrar la cinta. Ellos siempre sospechan de alguien que vaya a espiar.

(Descuelga el teléfono móvil).

EVA: Estoy ahora con los periodistas españoles, ¿está tranquilo todo ahí? Si por el camino pasa alguna cosa, me pueden avisar. Sí, disparos o podría entrar la policía. Entran a matar. La última vez mataron a 21 personas, del chiquitito hasta la abuelita.

REPORTERO: (Voz off) Aunque no nos han dejado grabarlo, dos jóvenes vigilan armados la entrada en la favela.

EVA: Ellos son la seguridad de la favela.

REPORTERO: ¿Es normal que estén siempre armados?

EVA: Sí, con metralletas, con escopetas... Podéis ver las marcas de bala en la pared. De combates entre la policía y los narcotraficantes. En Río mueren al año una media de 2.000 personas en situaciones violentas. Aquí, en Brasil se importan las armas entre Venezuela, Perú, Bolivia, Colombia... Por ejemplo cosas que hablo yo... otros jamás dirían... Es la vida en la favela, tiene sus reglas.

EVA: Una situación que nunca ha pasado, nunca han filmado a un narcotraficante sin permiso. Una vez descubrieron que un periodista estaba grabando con una cámara oculta. Lo mataron y lo cortaron en pedacitos.

(...)

EVA: Ella es la mamá de ellos. Parte de la ropa se lava a mano. Ella alquila la casa por unos 16 reales. El salario mínimo es de 400 reales. De comer tiene arroz, frijoles y pasta. No tiene frigorífico...

REPORTERO: ¿Recibe ayudas del gobierno?

MUJER: No.

REPORTERO: Y los que mandan aquí, ¿la ayudan?

EVA: Ehh.... No sé si lo voy a preguntar...

\footnotetext{
3 Programa de reportajes emitido en Cuatro en el que se denuncian todo tipo de problemas sociales y de actualidad.
} 

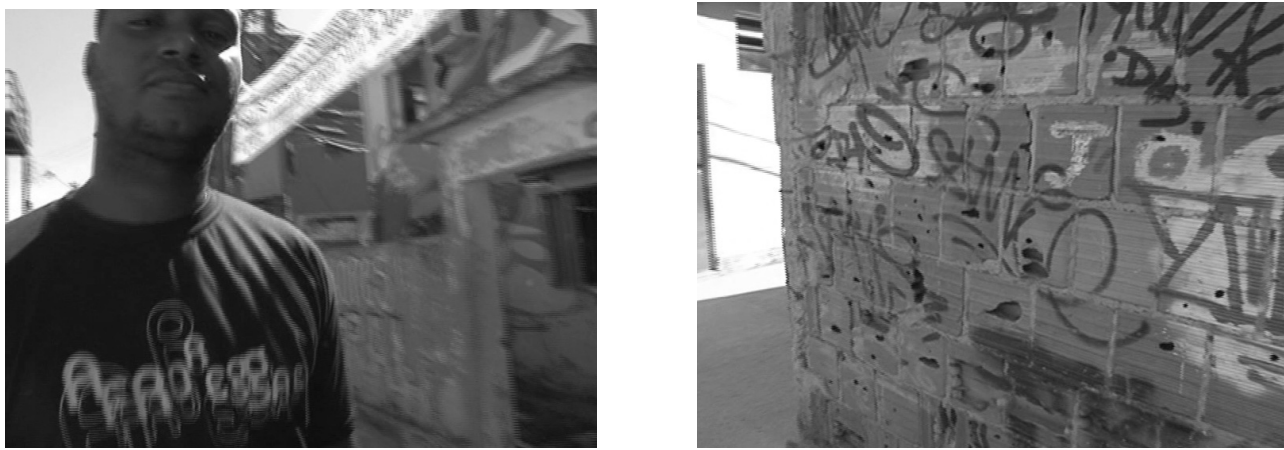

(1.2)

En este caso, el pensamiento vertical me sirvió para entrar en la favela Vigário Geral con la ONG AfroReggae, pero no para retratarla con libertad periodística. La persona de la primera fotografía (1.2) era nuestro guía y también nuestro censor. Aunque confesó que su hermano era narcotraficante, pero que él había dejado ese estilo de vida, controlaba cada persona que entrevistábamos. Si no le gustaba su testimonio nos recortaba el poco tiempo de grabación que teníamos.

El pensamiento vertical ofrece modelos normalizados para que el profesional pueda realizar su trabajo, pero al mismo tiempo le limita a ideas que están preestablecidas. En cambio, el pensamiento lateral se mueve en un terreno ambiguo y arriesgado que impulsa la creatividad del reportero para reflejar realidades sociales desde otras perspectivas. En Vigário Geral utilicé el pensamiento vertical y la sensación que tuve como profesional fue frustrante. Conseguimos permiso para grabar en una de las favelas más peligrosas de Brasil, pero lo que grabamos no reflejó por completo la realidad social que viven los ciudadanos de la favela.

La siguiente secuencia que voy a analizar pertenece al programa Callejeros, Viajeros: Montevideo. Varias zonas del barrio Villa del Cerro en Uruguay están consideradas como las más peligrosas de todo el país. De hecho, compañeros periodistas nos aseguraron que no podíamos entrar sin el respaldo de la policía. Si hubiéramos empleado el pensamiento vertical, el procedimiento hubiera sido diferente. Habríamos solicitado un permiso a la policía o a una ONG y habríamos grabado el barrio con las condiciones que ellos nos hubieran impuesto. Así jamás habríamos conocido las historias de algunas de las personas que entrevistamos. Desde gente humilde que vive con lo justo para sobrevivir hasta varios ex convictos que narran en primera persona las luchas de bandas armadas en su barrio. La pasta base, la resina de la cocaína, es también protagonista en la siguiente secuencia. Según el personaje entrevistado, sus enemigos la utilizan como moneda de cambio para que menores con adicción asesinen a cambio de una dosis de droga. Para conseguir retratar parte de esta realidad social, buscamos soluciones basadas en sistemas autoorganizados inspirados en el pensamiento lateral. O lo que Aladro (2011) denomina como un conocimiento intuitivo o de usabilidad, donde el autoaprendizaje, la iniciativa personal de búsqueda, creación y dinamización del mensaje, nos llevan a reclamar nuevas versiones del concepto de mensaje. 


\section{SECUENCIA VILLA DEL CERRO. MONTEVIDEO.}

MUJER: Yo, de la terminal del Cerro, estoy a ocho cuadras, unas diez de lo que es el Cerro marginal. A las afueras, al costado de donde están los marginados vive el presidente Pepe Mujica.

REPORTERO: ¿Cómo es el barrio?

MUJER: Es realmente peligroso.

POLICÍA: Es un barrio conflictivo como muchos de Montevideo. Si vas con una cámara y solo, se puede complicar.

MADRE: Mi hijo es aquel que está arriba.

HIJO: ¡Hay que luchar para comer!

MADRE: Picamos, tenemos que sacar los ladrillos. Lo limpiamos todo, sacamos todo lo que hay arriba y lo vendemos.

MUJER: No nos harán nada a la cámara, ¿verdad? Contigo.

MADRE: No, no... Muchos están aquí picando y se los llevan presos.

EX CONVICTO 1: Soy empresario. (Risas)

EX CONVICTO 2: Lo dejamos todo preparado, y vienen y se lo llevan, porque hay choques. Me querían disparar con un fierro. Me cogió y me puso contra la pared y me dijo: "ahora te voy a dar un tiro". Luego salí y le di un fierrazo. Después vino con un fierro y no me hizo nada. Ahora me tiene que matar.

REPORTERO: ¿Qué es un fierro?

EX CONVICTO 2: Un fierro es una cosa de tiros, una pistola. Estas (cicatrices) son de la cárcel. Estaba en la droga y la droga te lleva a robar.

MUJER: Son los que consideran más peligrosos y los meten en las peores zonas de la cárcel. La cárcel aquí en Uruguay es infrahumana.

REPORTERO: ¿Cómo sobrevive alquien en la cárcel?

EX CONVICTO 2: Sobreviviendo, sin tener que pagar comida, no hay que pagar agua. Lo único feo que tiene la cárcel es que no tenés libertad. Si te portas mal, la gente se porta mal contigo. Es mentira que te violan. Te violan si te dejás.

REPORTERO: En este barrio, ¿hay otra gente que venda droga?

EX CONVICTO 2: Nosotros no podemos hacer nada porque si hacemos algo envían a un menor con un arma, porque saben que no cumple condena.

REPORTERO: ¿Con qué le pagan?

EX CONVICTO 2: Le pagan con pasta base.

MUJER: La resina de la cocaína. Te hace necesitarla más rápido que la cocaína.

EX CONVICTO 2: Yo la consumí, yo sé lo que es. Esto es más seguro que una pistola. El cuchillo no falla la pistola sí. Puede venir una bala húmeda, puede encasquillarse. Es para proteger a mi familia, para nada más (coge el cuchillo y lo clava en un árbol). REPORTERO: ¿Ha fallecido alguien? ¿Algún conocido?

EX CONVICTO 2: Sí, una criatura de 5 meses aquí dentro. Aquí se crían 8 criaturas. REPORTERO: ¿Qué futuro hay para los niños?

EX CONVICTO 2: Acá, ninguno.

REPORTERO: ¿Qué les parece que el presidente del gobierno viva aquí cerca?

EX CONVICTO 2: No me llevo con esa gente. Todo es mentira lo que te dicen.

EX CONVICTO: Nosotros somos los peligrosos. Acá mandamos nosotros.

EX CONVICTO 2: Os hemos respetado, si no... 
La principal diferencia, según el enfoque de este artículo, entre el fragmento del barrio Villa del Cerro y el de la favela Vigário Geral es el tipo de pensamiento empleado. Mientras que en la secuencia de Rio de Janeiro predomina el uso del pensamiento vertical, un año después, en Montevideo, preferí someter a duda algunos modelos y probar con el pensamiento lateral. Según De Bono (1994: 215 y ss), la provocación es una de las bases del pensamiento lateral. Consiste en un experimento mental que rebasa los límites habituales de lo razonable establecidos por nuestra experiencia. En Montevideo decidimos realizar un experimiento al decidir entrar en una de las zonas más conflictivas de la ciudad sin el apoyo de una ONG, la policía o una importante institución. Creimos que la secuencia sería más honesta si contábamos la historia del barrio en contacto directo con la gente y sin intermediarios.

"La finalidad de la provocación es precisamente desviarnos del recorrido habitual del pensamiento. A partir de la provocación avanzamos hasta encontrar un nuevo punto que retrospectivamente, parece ventajoso. Tenemos que salir del mundo de la lógica para luego volver al él” (De Bono 1994: 220). La idea de grabar sin un respaldo institucional en una zona conflictiva fuera de España fue para mí una provocación, una idea alocada y arriesgada. Ante esta provocación establecimos racionalmente varias hipótesis con el objetivo de entrar en el barrio y ganarnos la confianza de sus habitantes.

Para analizar este proceso creativo voy a emplear la recopilación que hace Valbuena de la Fuente sobre las técnicas de creatividad en la Retórica Clásica: adicción, sustracción, sustitución y trastrocamiento. Utilizamos la adicción cuando decidimos añadir a nuestro grupo a una vecina del barrio, próxima a la zona marginal y convencerla para que nos acompañara altruistamente. Pensamos que una mujer uruguaya restaría desconfianza a la imagen de forasteros que transmitíamos. Personalmente emplee la sustitución al vestirme con la ropa menos llamativa que tenía. Los habitantes del barrio iban a ser los protagonistas de la historia, no yo. Por eso quería que se sintieran como tales. No quería que el tipo de ropa creara una barrera entre el reportero y los entrevistados. La sustitución también la empleé en el momento en el que entramos en el barrio. Sustituí el testimonio de la vecina que nos acompañaba por una nueva aliada. Busqué a la mujer del grupo para entablar conversación con ella. Coincidió ser la madre de varios jóvenes que acababan de salir de la cárcel (1.3). Sin la confianza que establecimos con ella, quizá sus hijos no nos hubieran respetado. Supongo que si hubiéramos intentado hablar directamente con los jóvenes, el encuentro no hubiera sido tan fluido. 

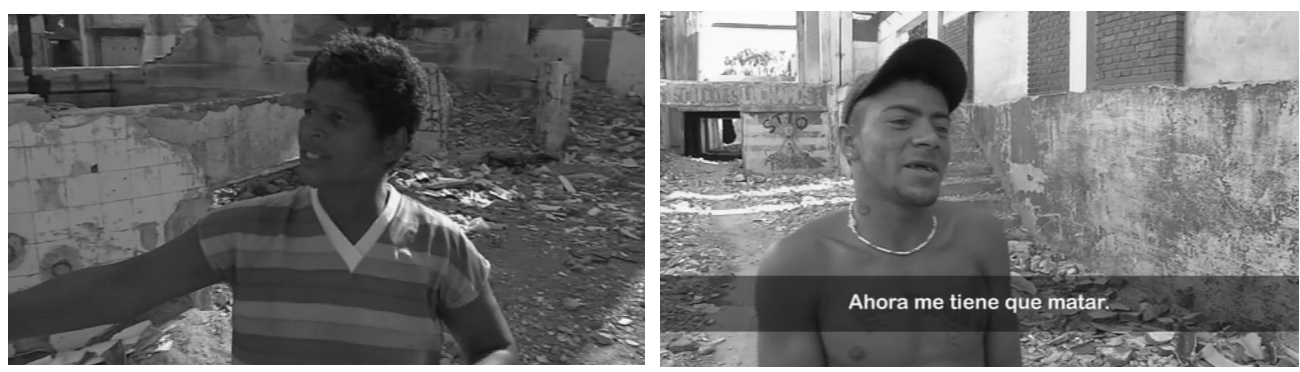

Por límites temporales y logísticos tuve que emplear la técnica de la sustracción. Es decir, acoté el estilo de vida del barrio a una única zona y a unos personajes determinados. Para mostrar diferentes aspectos de sus vidas, empleé la técnica del trastrocamiento. A lo largo de la secuencia cambiamos de temas. Desde el modelo de vida humilde en el barrio hasta los conflictos violentos.

A continuación transcribo el último fragmento que voy a analizar. Pertenece a una secuencia de la favela Ciudad de Dios ${ }^{4}$ en Rio de Janeiro. En este caso entramos en el barrio con una escuela de modelos de la comunidade ${ }^{5}$.

SECUENCIA CIUDAD DE DIOS. RIO DE JANEIRO.

REPORTERO: Ciudad de Dios es una película que rodó parte de sus escenas en la favela que lleva su nombre. Es la historia del crecimiento del crimen organizado en este suburbio de la ciudad en la época en la que el tráfico de drogas y la violencia impusieron su ley. Gisele, modelo de profesión, fundó junto a María Petersen la escuela de modelos que hay en la favela.

MARÍA: Todas las chicas están estudiando también. Las modelos están representando un talento dentro de Ciudad de Dios. Cambiar su vida no es salir de la favela, es tener una vida digna donde tú vives. Ellas han tenido críticas de varias revistas internacionales.

REPORTERO: Esta mujer es la madre de dos niñas que han ganado más de 2.000 euros en varios concursos de modelos y de baile.

MADRE: Soy ex presidiaria.

MARÍA: Ella era narcotraficante y estuvo en la cárcel tres años y medio, pero ella jamás consumió drogas.

REPORTERO: ¿Cuál es la esperanza de vida de un narcotraficante en Rio de Janeiro?

MARÍA: Es una vida que lleva a la muerte.

MARÍA: Ella salió y ahora se dedica a sus hijas... Esta escuela es una medida para quitar a los niños de la calle.

\footnotetext{
${ }^{4}$ Favela que es famosa internacionalmente por la película Cidade de Deus, de Fernando Meirelles.

${ }^{5}$ Eufemismo en portugués de la palabra favela.
} 
(...)

MARÍA: Ciudad de Dios estaba controlada por los traficantes de drogas. La policía entró en noviembre y hubo muchos tiroteos, yo inclusive escuché muchas batallas. Es una guerra que no tiene vencedores ni vencidos, porque la policía también está involucrada en el tráfico de drogas. Siempre la gente inocente es la que paga las consecuencias.

MUJER: A veces los antiguos narcotraficantes eran buenos, porque eran como Robin Hood nos daban lo que necesitabamos.

MARÍA: Ellos tenían un tipo de protección que ya no tienen.
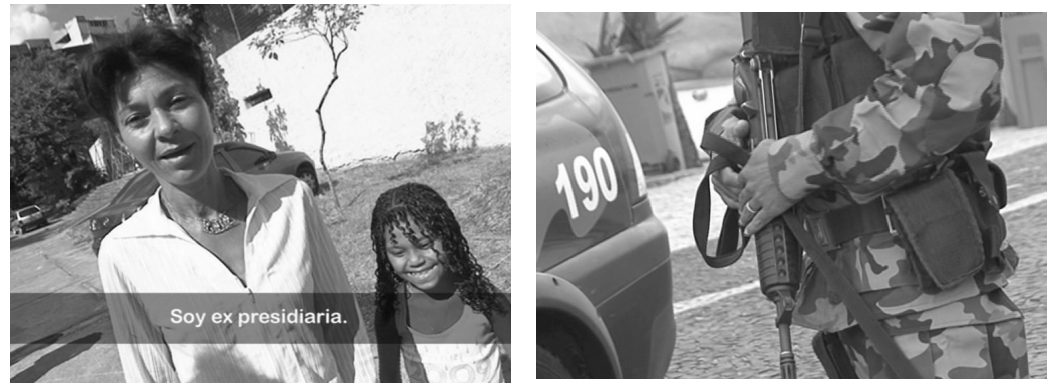

(1.4)

\section{CÁNONES DE CAUSALIDAD Y CREATIVIDAD}

Voy a analizar y comparar el fragmento de la favela Ciudad de Dios (Rio de Janeiro) con los dos anteriores que he expuesto en este artículo. Creo que es interesante incorporar a este análisis los cánones de causalidad del filósofo británico John Stuart Mill (1858) para formar ideas originales a partir de algunas circunstancias y fenómenos que se repiten en los diálogos de varias secuencias. El filósofo define los cánones de causalidad como un razonamiento inductivo que consiste en obtener conclusiones generales a partir de premisas que contienen datos particulares. Stuart Mill (1858: 229 y ss) enuncia cinco cánones de causalidad: canon de las concordancias, canon de las diferencias, canon conjunto de concordancia y diferencia, canon de las variaciones concomitantes y canon de los residuos.

El canon de los residuos enuncia lo siguiente: si se cercena de un fenómeno aquella parte de la que se sabe por previas inducciones que es el efecto de ciertos antecedentes, el residuo del fenómeno es el efecto de los antecedentes restantes (Stuart Mill 1858: 229). En el principio de la secuencia de la barriada de Montevideo empleé el canon de los residuos. Había comprobado en reportajes anteriores de España que las madres de otras zonas marginales son muy respetadas por sus hijos y por los supuestos criminales del barrio. Establecí la hipótesis de que si conseguía la confianza de una madre tendría acceso a sus hijos y al resto de los jóvenes de la barriada sin necesidad de ir acompañado por una ONG. En este caso el fenómeno sería el retrato de una zona conflictiva. El efecto consistiría en la búsqueda de una madre en el barrio. El residuo del canon establecería que si un reportero consigue ganarse la confianza de una madre en un barrio marginal tendrá acceso al resto de 
sus habitantes. Para Valbuena de la Fuente, (1998) el empleo de los cánones de causalidad es una de las herramientas más poderosas de la creatividad en las negociaciones que permite encontrar lo que el autor denomina como el ruido de la información, y ayuda a establecer hipótesis útiles. Para un reportero, estas herramientas pueden ser válidas para conseguir imágenes y testimonios exclusivos sobre denuncias sociales.

El canon de las concordancias manifiesta lo siguiente: si dos o mas casos del fenómeno sometido a investigación tienen sólo una circunstancia en común, la circunstancia en la que únicamente todos los casos concuerdan es la causa o efecto del fenómeno dado (Stuart Mill 1858: 222). Como reportero he empleado este método decenas de veces en un caso concreto. En todas las zonas marginales que he grabado me he percatado de que hay una circunstancia en común que está relacionada con la Teoría de los Juegos Comunicativos de Eric Berne. Los habitantes con menos recursos económicos suelen participar en los juegos: No es horrible y Guardias y ladrones (Valbuena (ed.) 2006: 182 y ss). El reportero y los entrevistados conectan entre sí porque participan en los mismos juegos. Una parte quiere mostrar sus desgracias y sus fechorías y la otra quiere grabar esos aspectos de la realidad. Las necesidades de los juegos comunicativos forman parte de la circunstancia común entre varios fenómenos sometidos a grabación. Es decir, cuando un reportero acude a una zona marginal sabe que esos juegos comunicativos son causas que le permiten grabar en zonas conflictivas, donde, en algunos casos, ni la policía puede entrar. Esa capacidad creativa, consciente o instintiva, del reportero evita posibles conflictos violentos y construye negociaciones cooperativas entre ambos bandos: periodista y entrevistado.

Los cánones de causalidad son sistemas autoorganizados similares a los que propone De Bono con el pensamiento lateral (1994). Los cánones de Mill funcionan como modelos abiertos donde un reportero puede relacionar, bajo su perspectiva, las diferentes circunstancias y fenómenos que intervienen durante una grabación. Stuart Mill (1858: 228) define el canon de las diferencias de la siguiente manera: si un caso en el que el fenómeno sometido a investigación ocurre, y un caso en el que no ocurre, tienen todas sus circunstancias en común, salvo una. Y ésta una ocurre en solo el primero, la circunstancia en la que únicamente los dos casos diferen, es el efecto, o la causa, o una parte indispensable de la causa del fenómeno. Voy a comparar los diálogos referentes a la declaración de delitos en los fragmentos de Ciudad de Dios y de Villa del Cerro. El fenómeno es la confesión de detalles sobre una historia delictiva. El primer caso es una mujer brasileña con una intérprete y el segundo fragmento es un hombre uruguayo sin intérprete. La diferencia entre ambas circunstancias puede ser por dos motivos. Que cuando hay un intérprete entre el entrevistado y el reportero se pierden muchos de los detalles de la historia o que las mujeres son menos propicias a contar detalles de actos delictivos cuando está sus hijas a su lado.

El canon conjunto de concordancias y diferencias manifiesta lo siguiente: si dos o más casos en los que el fenómeno ocurre tienen solo una circunstancia en común, mientras dos o más casos en los que éste no ocurre no tienen nada en común, salvo la ausencia de esa circunstancia, la circunstancia en la que únicamente las dos series 
de casos difieren es el efecto, o la causa, o una parte necesaria de la causa del efecto (Stuart Mill 1858: 227). Además de las secuencias que he mostrado en este artículo voy a incluir la secuencia que grabamos en la favela Nueva Ciudad ${ }^{6}$. Los dos primeros casos son las favelas de Ciudad de Dios y la barriada de Montevideo. El fenómeno es la grabación de un testimonio en primera persona de un ex traficante de drogas. La circunstancia en común es la ausencia de ONGs u otras instituciones. Los dos segundos casos son la secuencia en la favela Vigário Geral y en la favela Nueva Ciudad. Aquí el fenómeno no ocurre. No grabamos testimonios de ex traficantes de droga. La circunstancia en común es la presencia de ONGs y/o otras instituciones. Por lo que la circunstancia en la que las dos series de casos difieren es el efecto, o la causa, o una parte necesaria de la causa del efecto. En este caso la presencia de representantes, tanto de una ONG como de la Iglesia, provoca que sea más difícil indagar sobre los problemas de narcotráfico en los lugares citados. Esto propicia que tengamos que centrar la grabación únicamente en una serie de problemas sociales relacionados con la labor que realizan dichas organizaciones.

El canon de las variaciones concomitantes (Stuart Mill 1858: 234) enuncia que todo fenómeno que varía de alguna manera, cuando otro fenómeno varía de una cierta manera particular, es o una causa, o un efecto de ese fenómeno, o se liga con él por algún lazo causal. Cuando los criminales de una zona marginal pasan de estar desorganizados a configurar una estructura sólida se produce la variación de un fenómeno que causa un efecto en otro fenómeno. Ese cambio obliga a los periodisticas a aceptar las normas que impongan los narcotraficantes para grabar en sus favelas. Cuanto más organizado esté una zona marginal, más difícil será retratar su realidad social con libertad periodística.

\section{CONCLUSIONES}

En este artículo no he pretendido crear una serie de axiomas universales sobre cómo debe actuar un reportero en una zona conflictiva de América Latina. Todo lo contrario. Cuando se acepta un modelo como válido sin someterlo a duda estamos en el terreno de las creencias, en el pensamiento anti-creativo. "Las ideas se tienen; en las creencias se está. Pensar en las cosas o contar con ellas" (Ortega y Gasset 1945: 15). En este artículo he analizado una pequeña parte del proceso creativo que empleé en la búsqueda de ideas útiles para algunos de mis reportajes. Pero al ser un análisis personal, basado en sistemas autoorganizados, estas ideas no pueden servir como modelos normalizados. Sin embargo, el análisis de los ejemplos expuestos demuestra el poder que tiene el pensamiento creativo, frente a la aceptación de los modelos normalizados, cuando un reportero quiere retratar zonas difíciles de grabar.

Es indudable que esa creatividad inicia un proceso comunicativo, intencionado o desinteresado, para impulsar el desarrollo de las zonas retratadas. En los ejemplos analizados se confirma la hipótesis de que el reportero que emplea un pensamiento

${ }^{6}$ Favela de Rio de Janerio que acudimos con el apoyo de un representante de la Iglesia Católica. 
creativo tiene más posibilidades de descubrir realidades sociales e historias desconocidas para sus espectadores. La duda, la provocación y otras técnicas creativas ayudan al periodista a buscar alternativas y soluciones para conseguir mostrar zonas de difícil acceso para cualquier persona ajena al barrio. La creatividad se convierte en el primer paso del proceso comunicativo entre el periodista y sus espectadores. Esa información contribuye a concienciar a la opinión pública sobre una serie de problemas sociales. A mi juicio, el proceso analizado en este artículo sienta las bases que impulsan la primera fase del futuro desarrollo de lugares marginales. Este primer impulso difunde una realidad social a un fragmento de la sociedad. Sin el conocimiento no puede existir el desarrollo. En cualquier caso, cualquiera de las ideas que desarrollo en este artículo es susceptible de convertirse en una creencia o de someterse a duda por el lector.

\section{REFERENCIAS BIBLIOGRÁFICAS}

Aladro, E. (2011): "La teoría de la información ante las nuevas tecnologías de la comunicación". Revista CIC (Cuadernos de Información y Comunicación), 16.

CASTAÑARes, W. (2006): La televisión moralista. Madrid. Fragua.

De Bono, E. (1994): El pensamiento creativo. Barcelona. Paidós plural.

KAPUSCINSKI, R (2002): Los cínicos no sirven para este oficio. Barcelona. Anagrama.

Lipman, M. (1991): Pensamiento complejo y educación. Madrid. Ediciones de la torre.

Ortega y Gasset, J. (1945): Ideas y creencias. Argentina. Espasa Calpe.

-(2005): Ideas sobre el teatro y la novela. Madrid. Alianza Editorial.

Pulitzer, J (2011): Sobre el periodismo. España. Gallo Nero.

RANDALL, D. (2008): The universal journalist. London: Pluto press.

StenBerg, Richard (1999): Handbook of Creativity. Cambridge. Cambridge University Press.

StUART Mill, J. (1858): A system o logic, ratiocinative and inductive. New York. Harper $\&$ Brothers.

Valbuena, F (ed.) (2006): Eric Berne, teórico de la comunicación. Madrid. Edipo.

- (1998-1999): "Retórica y Poética en los relatos del Padre Brown de G.K. Chesterton". Revista CIC (Cuadernos de Información y Comunicación), 4. 


\title{
RESUMEN
}

Cuando un reportero realiza un reportaje televisivo en una zona conflictiva puede escoger dos caminos: grabarlo según modelos establecidos anteriormente o buscar nuevos modelos creativos. En este artículo analizo mi perspectiva personal como reportero en varios barrios marginales de América Latina y los métodos creativos que empleé para poder retratarlos. La creatividad forma parte de un proceso comunicativo que contribuye al desarrollo de las zonas grabadas. El pensamiento creativo hace posible que el reportero pueda mostrar realidades sociales a los espectadores de su programa. De esta forma, el periodista contribuye en la primera fase del desarrollo de dichas zonas con la difusión y el conocimiento por parte de la opinión pública de una serie de problemas sociales.

Palabras clave: Creatividad, desarrollo, reportero, comunicación, idea, creencia.

\begin{abstract}
When a reporter is making a TV documentary about a dangerous neighborhood can take two ways: the established model or the creative one. On this article I analyze my personal point of view as a reporter in several neighborhoods of Latin America and the creative methods I used to this works. Creativity is a part of the communicative process that can help to the development of this zones. The creative thinking makes possible that the reporter can show social realities to the channel's audience. Actually, the journalism help to the first step of development in this marginal places through the broadcast and the consciousness-raising of the public opinion of this social problems.
\end{abstract}

Keywords: Creativity, develoment, reporter, communication, idea, belief.

\section{RÉSUMÉ}

Quand un journaliste a fait un documentaire sur un quartier dangereux peut prendre deux formes: l'enregistrement objectif ou la créativité. Dans cet article, $\mathrm{j}$ 'analyse mon point de vue personnel en tant que journaliste dans plusieurs quartiers de l'Amérique latine et les méthodes créatives que j'ai utilisé. La créativité est une partie du processus de communication qui peut aider au développement de ces zones. La pensée créative permet que le journaliste montre les réalités sociales à son public. En fait, le journalisme aide à la première étape de développement dans ces lieux marginaux à travers la diffusion et la sensibilisation de l'opinion publique sur ces problèmes sociaux.

Mots clé: Créativité, developpement, journaliste, communication, idée, croyance. 